\title{
Extraction of the Muon Signals Recorded with the Surface Detector of the Pierre Auger Observatory Using Recurrent Neural Networks
}

\author{
Juan Miguel Carceller ${ }^{a, *}$ on behalf of the Pierre Auger $^{b}$ Collaboration \\ (a complete list of authors can be found at the end of the proceedings) \\ ${ }^{a}$ University of Granada and C.A.F.P.E, Granada, Spain \\ ${ }^{b}$ Observatorio Pierre Auger, Av. San Martín Norte 304, 5613 Malargüe, Argentina \\ E-mail: spokespersons@auger.org, auger_spokespersons@fnal.gov
}

\begin{abstract}
We present a method based on the use of Recurrent Neural Networks to extract the muon component from the time traces registered with water-Cherenkov detector (WCD) stations of the Surface Detector of the Pierre Auger Observatory. The design of the WCDs does not allow to separate the contribution of muons to the time traces obtained from the WCDs from those of photons, electrons and positrons for all events. Separating the muon and electromagnetic components is crucial for the determination of the nature of the primary cosmic rays and properties of the hadronic interactions at ultra-high energies.

We trained a neural network to extract the muon and the electromagnetic components from the WCD traces using a large set of simulated air showers, with around 450000 simulated events. For training and evaluating the performance of the neural network, simulated events with energies between $10^{18.5} \mathrm{eV}$ and $10^{20} \mathrm{eV}$ and zenith angles below 60 degrees were used. We also study the performance of this method on experimental data of the Pierre Auger Observatory and show that our predicted muon lateral distributions agree with the parameterizations obtained by the AGASA collaboration.
\end{abstract}

$37^{\text {th }}$ International Cosmic Ray Conference (ICRC 2021)

July 12th - 23rd, 2021

Online - Berlin, Germany

\footnotetext{
*Presenter
} 

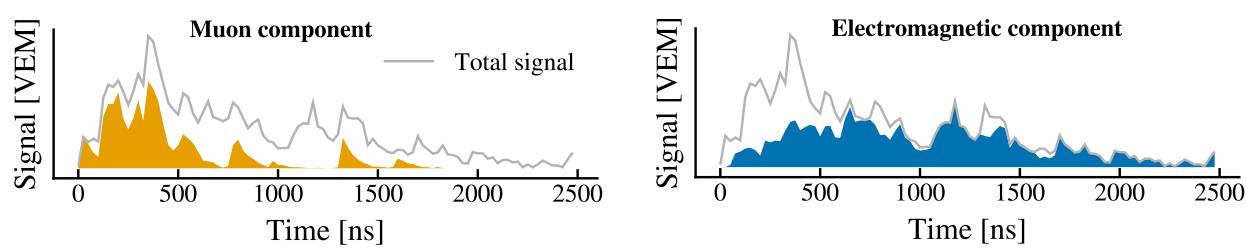

Figure 1: Signal in time measured by a single station of the SD for a simulated event. The total signal corresponds to the signal measured by the SD and is the sum of the muon component (left) and the electromagnetic component (right).

\section{Introduction}

Ultra-high-energy cosmic rays (UHECRs) are atomic nuclei with energies above $10^{18} \mathrm{eV}$. The Pierre Auger Observatory was designed to unveil the origin of these particles. It is the largest cosmicray detector built so far and comprises an area of $3000 \mathrm{~km}^{2}$. It is a hybrid detector featuring two techniques: measuring the fluorescence light emitted by atmospheric nitrogen with the Fluorescence Detector and measuring signals left by secondary particles of the shower that reach the ground with the Surface Detector (SD). This work focuses on the data collected with the SD, which operates with a duty cycle close to $100 \%$. Particles traverse the 12000 litres of ultra-pure water contained in each station of the SD and the emitted Cherenkov photons are collected by three photomultiplier tubes (PMTs). The resulting signal is proportional to the sum of the Cherenkov radiation in water produced by electromagnetic particles (photons, electrons and positrons) and muons, see Figure 1. Muons are highly penetrating particles, therefore they usually arrive at the stations at earlier times than the electromagnetic particles. Furthermore, they produce a signal that is spiky, in contrast to the signal from electromagnetic particles, which is more spread in time.

The determination of the muon component in the air shower is crucial to infer, for each event, the mass of the primary particle, which is a key ingredient in the searches conducted to pinpoint the sources of UHECRs. However, with the current design of the SD, the separation of the muon and electromagnetic components can only be done for events with large zenith angles or for distances far off from the shower core, see for example [1]. Hence, for most of the recorded data, this separation cannot be done in a straightforward way. In this work, starting from the total time trace, we estimate the muon signal for each WCD as a function of time using machine learning techniques.

\section{The method}

Our approach is based on Recurrent Neural Networks (RNNs). RNNs are specially well-suited for time series due to their memory mechanism.

\subsection{The input}

The traces recorded by the water-Cherenkov detectors of the SD are the input to the neural network. The electronics sample the signals in bins of $25 \mathrm{~ns}$. The calibrated signals are expressed in Vertical Equivalent Muons (VEMs), which correspond to the most-likely signal deposited by a muon that vertically traverses the center of the detector. The traces used in this study are obtained after averaging the traces of every functioning PMT in a WCD. 


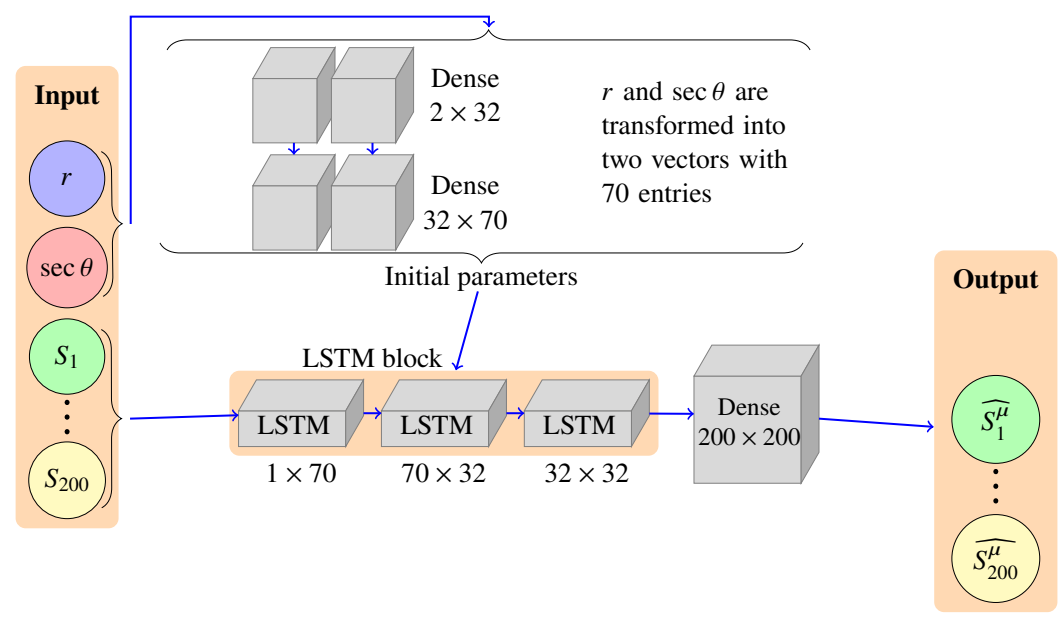

Figure 2: Schematic drawing of the input, architecture and output of the neural network.

We use the first 200 bins $(5 \mu \mathrm{s})$ of each trace. Traces that are shorter than 200 bins are padded with zeros at the end. The choice is motivated because the most relevant information is encapsulated in the first 200 bins of each trace. However, the trace information is not enough to accurately determine the muon component of extensive air showers. The amount of atmosphere that the particles traverse plays an important role, since the electromagnetic and muon components are attenuated differently in the atmosphere. For this reason, we added two more input parameters: the secant of the reconstructed zenith angle, $\sec \theta$, and the distance to the core on the plane perpendicular to the shower $r$ [2].

\subsection{Recurrent Neural Network (RNN) architecture}

The architecture of the neural network is shown in Figure 2. The input is a vector of 202 components: $r, \sec \theta$ and the 200 values of each trace $S_{1}, S_{2}, \ldots, S_{200}$, where $S_{i}$ is the value of the signal measured at the time bin $t_{i}$. A complete description of the architecture and the operations that are performed can be found in ref. [3]. The model has a total of 87212 trainable or free parameters.

\subsection{Data selection and training}

The neural network is trained with a library of simulated showers, with the same number of showers initiated by protons, helium, oxygen and iron nuclei. The energy range covered by simulations spans from $10^{18.5} \mathrm{eV}$ to $10^{20.2} \mathrm{eV}$ for zenith angles up to $60^{\circ}$. Each shower is simulated

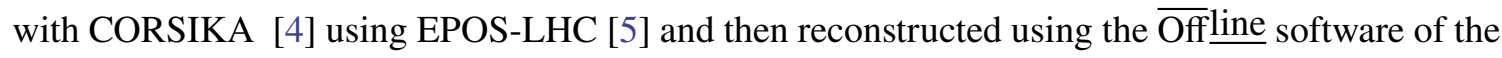
Pierre Auger Collaboration [6]. We select events that fulfill the quality condition that the station measuring the highest signal has to be surrounded by six operating neighbours, thus avoiding events that fall at the edges of the array.

Around 450000 simulated events were available. The events were sampled randomly and assigned to the training, validation or test data sets using a uniform distribution in energy and $\sec \theta$ for the validation and test sets; the remaining events were assigned to the training set. A complete description of the parameters, loss function and optimizer used for training can be found in ref. [3]. 

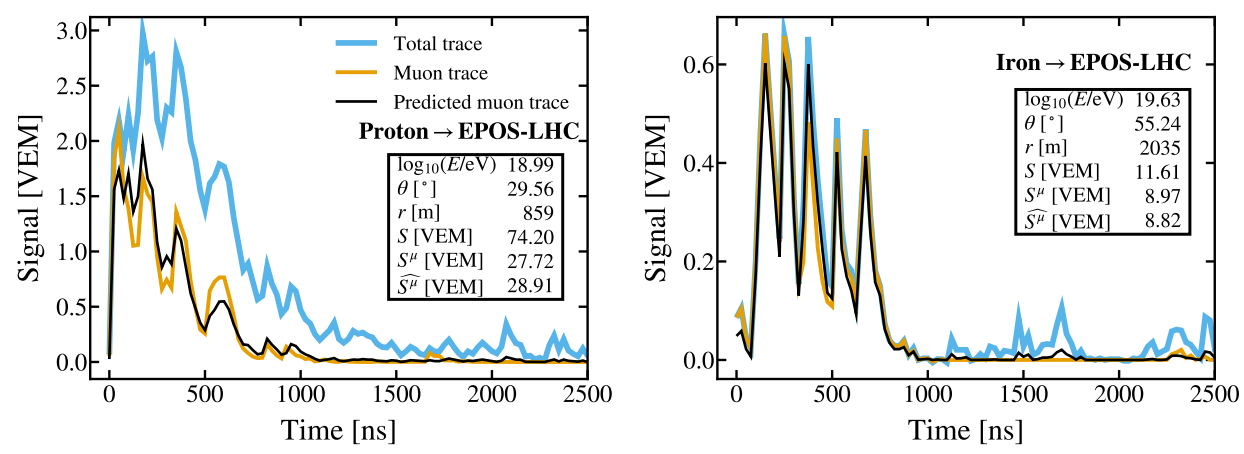

Figure 3: Examples of predicted muon traces for two simulated events with EPOS-LHC, for a electromagnetic-dominated signal (left) and muon-dominated signal (right). The prediction (black line) agrees well with the shape of the simulated muon trace (orange line). The blue thicker line corresponds to the signal measured by a WCD.
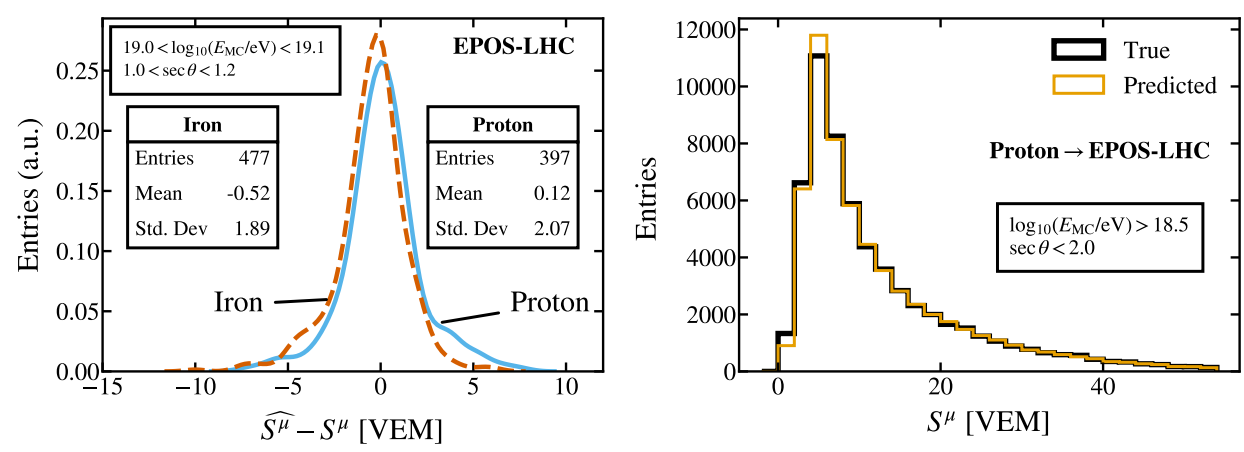

Figure 4: Left: Distribution of the difference between the integral of the predicted muon signal $\widehat{S^{\mu}}$ and the integral of the true muon signal $S^{\mu}$. Right: Distribution of $\widehat{S^{\mu}}$ and $S^{\mu}$ for all the stations in the test set.

\section{Performance on simulations}

We show examples of the predictions of the neural network in Figure 3. As a general comment, we observe that, qualitatively, the prediction follows the shape and peaks of the total signal. The network has learnt to reproduce the main features of the muon trace.

\subsection{Integrals of the trace}

One way to assess the performance of the method is to compute the integral of the predicted muon trace, $\widehat{S^{\mu}}=\sum_{i=1}^{200}{\widehat{S^{\mu}}}_{i}$ and compare it to the integral of the true muon trace $S^{\mu}=\sum_{i=1}^{200} S^{\mu}{ }_{i}$. The integral of the muon trace is is related to the total number of muons that reach the ground. In the left panel of Figure 4, we show the difference between $\widehat{S^{\mu}}$ and $S^{\mu}$ for a particular bin of energy and zenith angle for protons and iron nuclei. The difference is compatible with zero and does not show a strong dependence with the value of the true muon signal. In the right panel of Figure 4, we show the distribution of $\widehat{S^{\mu}}$ and $S^{\mu}$ for all the WCDs in the test set for showers initiated by a proton primary. The two distributions have similar shapes.

In Figure 5, the performances of the method are shown as a function of the primary energy. In the left panels, the mean value and standard deviation of the difference between the predicted 

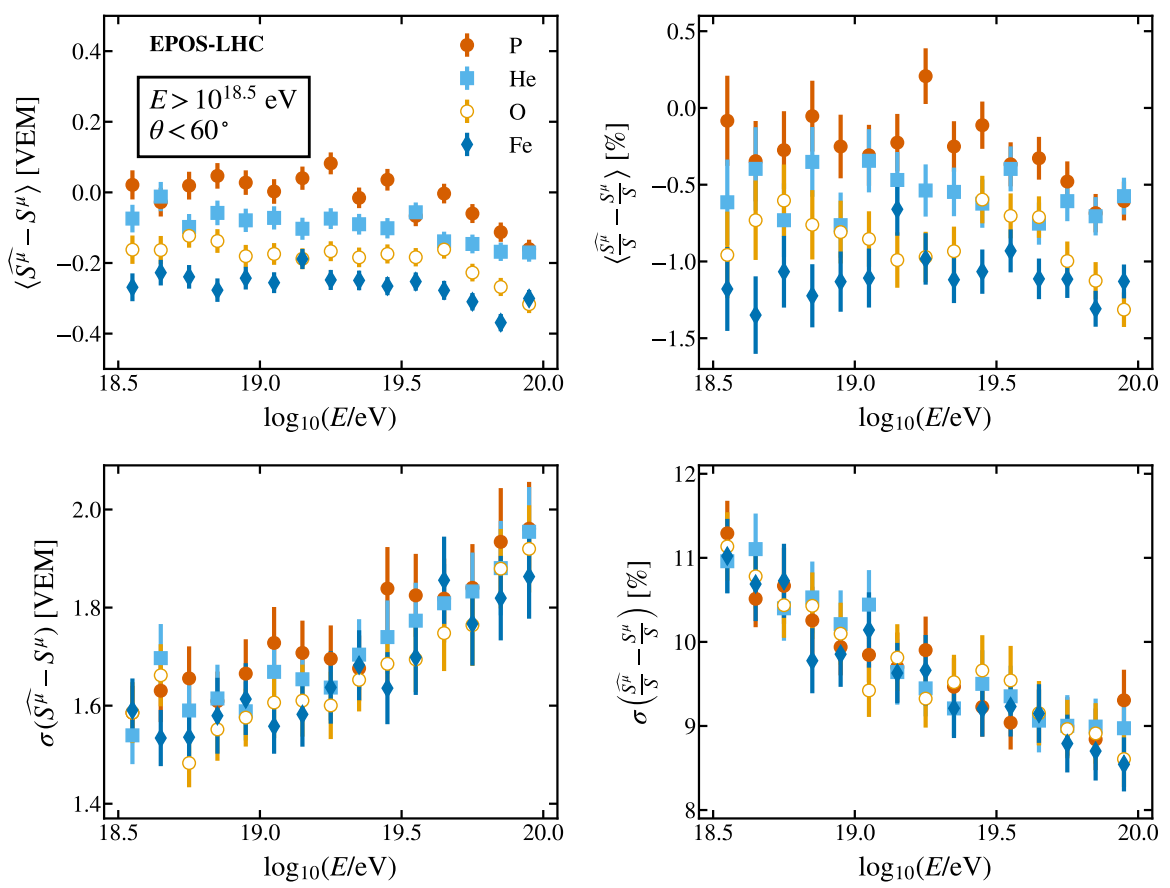

Figure 5: Left: Mean value (top) and standard deviation (bottom) of the difference between the predicted and values of the true muon signal as a function of energy. Right: Relative bias (top) and resolution (bottom) for the determination of the muon fraction as a function of the energy.
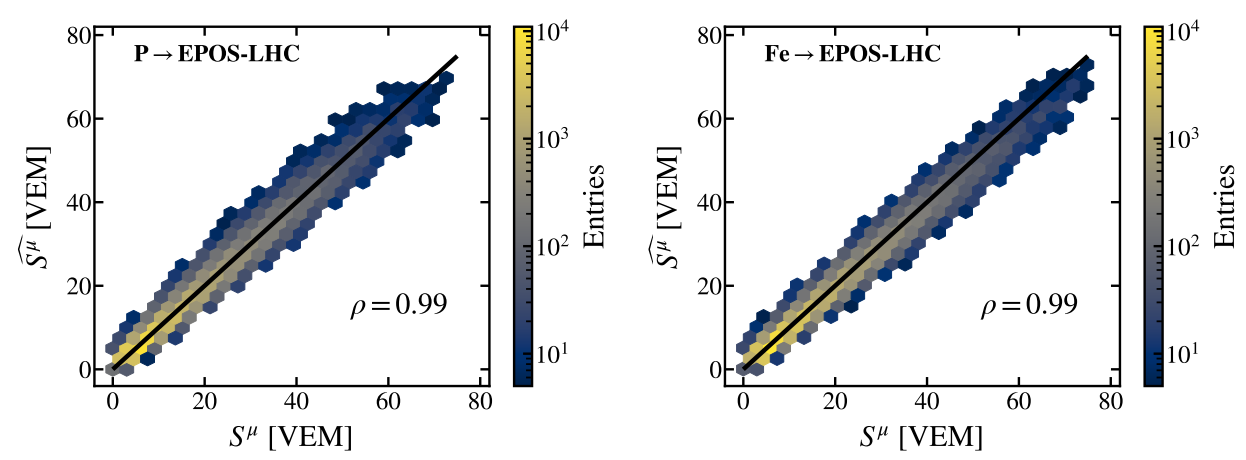

Figure 6: Integral of the predicted muon trace as a function of the integral of the true muon trace. The black line corresponds to a perfect prediction and $\rho$ is the Pearson correlation coefficient.

and true values are shown for the different primaries. The mean values are close to zero and the standard deviation is less than 2 VEMs in most bins. These performances readily translate into the biases and resolutions for the muon fraction shown in the right panels. The biases are within $2 \%$ for all the energies and angles explored here, regardless of the primaries. The resolutions are of the order of $11 \%$ at $10^{18.5} \mathrm{eV}$, improving with increasing energies.

The performance of the method can also be evaluated as a function of the simulated muon signal $\left(S^{\mu}\right)$. In Figure 6, we show the density plot of the predictions and the true values. The predictions are highly correlated with a Pearson correlation coefficient that is 0.99 for all primaries. 

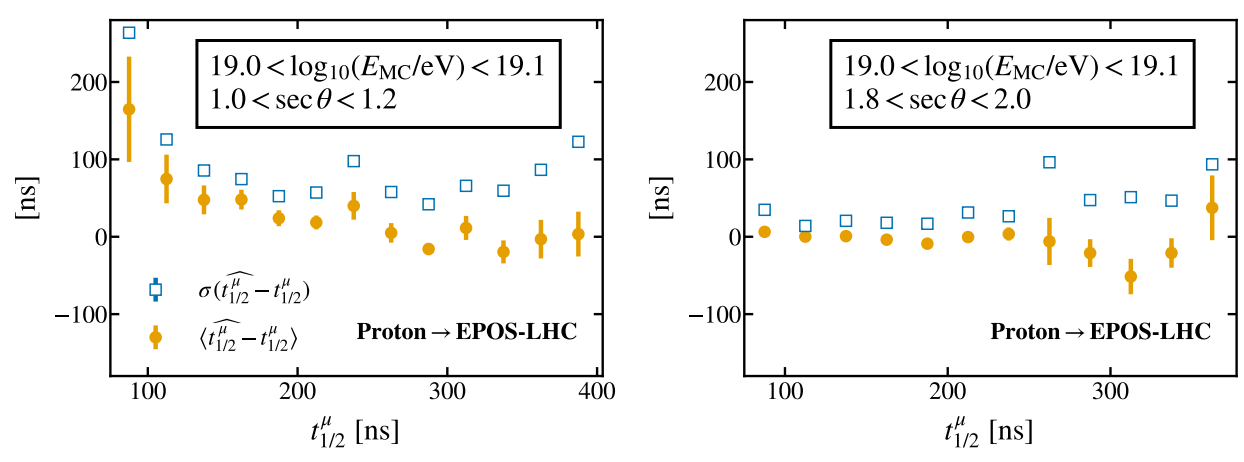

Figure 7: Mean and standard deviation of the difference between the risetime of the predicted muon signal $\widehat{t_{1 / 2}^{\mu}}$ and the risetime of the true muon signal $t_{1 / 2}^{\mu}$ for all the stations from events with the energies and zenith angles specified in the boxes.

For vertical events, with $S^{\mu}>5$ VEM, the resolution in the determination of the muon signal for each WCD is about $20 \%$ reducing to $10 \%$ for events with $\sec \theta \simeq 2.0$.

\subsection{Hadronic interaction model}

We have trained our neural network on events simulated with EPOS-LHC. We have also tested the predictions of this neural network on simulations done with different hadronic models. For simulations produced using QGSJetII-04 [7], the performance is similar to those presented with EPOS-LHC. Similar results are also obtained when testing the predictions on simulations done with Sibyll 2.3 [8]. With these results we show that the neural network predictions rely on the response of the detectors to muons and are independent of the hadronic interaction model.

\subsection{Risetime of the muon signal}

The risetime $t_{1 / 2}$ is defined as the difference between $t_{10}$, the time corresponding to the first time bin where the integral of the signal reaches $10 \%$ of its total value, and $t_{50}$, the time where it reaches $50 \%$. The muon component has a smaller risetime than the electromagnetic component since muons arrive earlier and in a shorter window of time.

In Figure 7, we compare the risetime of the predicted muon trace $t_{1 / 2}^{\widehat{\mu}}$ with the risetime of the simulated muon trace $t_{1 / 2}^{\mu}$. The standard deviation is less than $100 \mathrm{~ns}$ ( 4 time bins) for most values of the true risetime. This is small compared to the risetime of the total signal, which has a mean of $500 \mathrm{~ns}$ for the left panel of Figure 7. While there is a larger bias at lower risetimes in the left panel, vertical showers with such a short risetime $\left(t_{1 / 2}^{\mu}<100\right)$ trigger less than $1.5 \%$ of the stations included in that bin. The mean value approaches zero and the performance improves as the zenith angle increases. This means that the network successfully predicts the shape of the muon trace.

\section{Comparison to data}

In this section we assess how the neural network performs when applied to experimental data. The sample of events selected has the same cuts as the simulations used for training. In total, there are 177000 events registered from 2004 to 2017. In Figure 8, we show examples of the muon trace predicted for two typical signals recorded with two independent WCDs. 

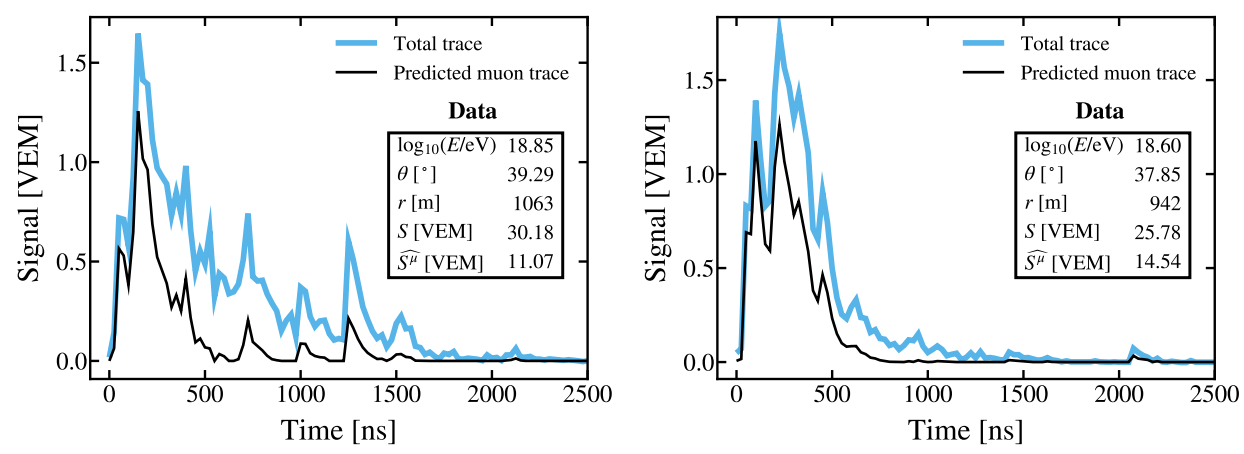

Figure 8: Examples of the predicted muon traces for two WCDs that belong to two different events recorded by the SD.
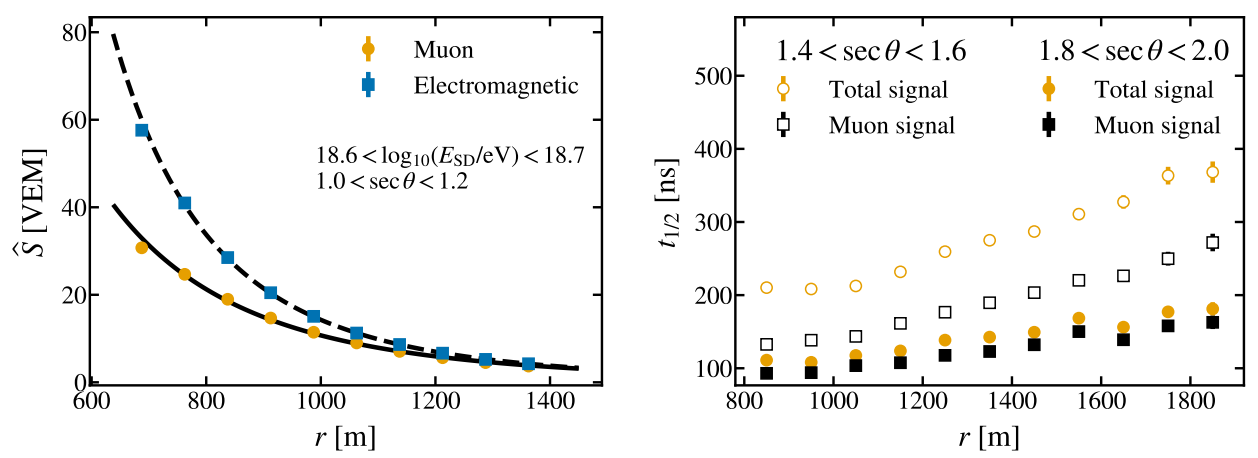

Figure 9: Left: Lateral distribution of the predicted muon and electromagnetic components for data. The solid and dashed lines are the outcome of two independent fits (see text for details). Right: Measured risetime of the total signal and risetime of the predicted muon signal as a function of the distance for two different zenith angle ranges.

A straightforward way to verify the robustness of the muon signal measurement is to study the behaviour of the total muon signal as a function of the distance to the air-shower axis, i.e. the muon lateral distribution function (LDF). The muon LDF is illustrated in Figure 9, together with the electromagnetic LDF. For each WCD, we can obtain the electromagnetic component simply by subtracting the predicted muon signal from the total recorded signal.

In addition, we compare our data to the parameterizations of the LDF of muon and electromagnetic particles that best fit the Akeno measurements. The authors of ref. [9] studied the properties of muons above $1 \mathrm{GeV}$ at different distances to the shower core for events with $\sec \theta<1.2$. The behaviour of the Akeno data is well reproduced by the function proposed by Greisen in ref. [10], Eqs. (4) and (6) in ref. [9]. We fit our data with those expressions and the parameters that best reproduce Akeno data, just leaving the overall normalization free. The outcome of the fit is shown as a solid line in the left panel of Figure 9 for the energy range $10^{18.6} \mathrm{eV}$ to $10^{18.7} \mathrm{eV}$. The level of agreement between our measurements and the muon lateral distribution function extracted from Akeno data is remarkable.

In ref. [11], the Akeno data are used to extract a parameterization of the density of the 
electromagnetic signal as a function of the distance to the shower core (Eq. (2.1) in ref. [11]). We use that parameterization to fit our predicted electromagnetic signal, leaving the overall normalization free as well as the parameter referred to as $\eta$ in the original publication. As for the case of muons, the agreement with the Akeno parameterization of the electromagnetic signals is very good (dashed line in Figure 9).

The data sample was also used to carry out a cross-check involving the arrival times of the particles: we compared the risetimes for the total and predicted muon signals as function of the distance to the core for two different zenith angle bins, see the right panel of Figure 9. As expected, the muon risetime is consistently smaller since muons arrive earlier. As the zenith angle increases, the fraction of the muon signal grows, thus the risetime of the total signal and the muon risetime become more similar. This behaviour is correctly reproduced by the predictions of the RNN.

\section{Summary}

We have shown that a Recurrent Neural Network is a suitable tool to predict the muon content of the time traces measured with each WCD of the SD of the Pierre Auger Observatory. The neural network is trained on simulations, but the predictions are independent of the model used to simulate hadronic interactions. The muon signal for each WCD can be measured with a resolution that decreases from $20 \%$ to $10 \%$ as the zenith angle increases. Likewise, the muon fraction with respect to the total signal is estimated with biases that are within a $2 \%$ and a resolution that is lower than $11 \%$. The muon risetime can be predicted with a standard deviation usually lower than $100 \mathrm{~ns}$, which tells us that the shape of the signals is well predicted.

When applied to data, the behaviour of the extracted muon and electromagnetic signals agrees well with relevant measurements found in the literature while other observables, such as the risetime, follow the behaviour dictated by basic physics principles.

\section{References}

[1] Pierre Auger Collaboration, Phys. Rev. D 91 (2015) 032003 [1408. 1421].

[2] Pierre Auger Collaboration, JINST 15 (2020) P10021 [2007.09035].

[3] Pierre Auger Collaboration, Accepted for publication in JINST (2021) [2103.11983].

[4] D. Heck, J. Knapp, J. Capdevielle, G. Schatz and T. Thouw, FZKA-6019 (1998) .

[5] T. Pierog et al, Phys. Rev. C 92 (2015) 034906 [1306.0121].

[6] Pierre Auger Collaboration, 2005 IEEE Nuclear Science Symposium and Medical Imaging Conference 2 (2005) 1000 [astro-ph/0601016].

[7] S. Ostapchenko, Phys. Rev. D 83 (2011) 014018 [1010 . 1869].

[8] E.-J. Ahn et al, Phys. Rev. D 80 (2009) 094003 [0906 . 4113].

[9] AGASA Collaboration, J. Phys. G 21 (1995) 1101.

[10] K. Greisen, Annual Review of Nuclear Science 10 (1960) 63.

[11] M. Nagano et al, J. Phys. G 18 (1992) 423. 


\section{The Pierre Auger Collaboration}

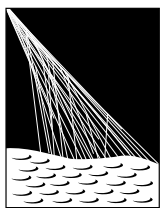

PIERRE

P. Abreu ${ }^{72}$, M. Aglietta ${ }^{54,52}$, J.M. Albury ${ }^{13}$, I. Allekotte ${ }^{1}$, A. Almela $^{8,12}$, J. Alvarez-Muñiz ${ }^{79}$, R. Alves Batista ${ }^{80}$, G.A. Anastasi ${ }^{63,52}$, L. Anchordoqui ${ }^{87}$, B. Andrada ${ }^{8}$, S. Andringa ${ }^{72}$, C. $A_{r a m o}{ }^{50}$, P.R. Araújo Ferreira ${ }^{42}$, J. C. Arteaga Velázquez ${ }^{67}$, H. Asorey ${ }^{8}$, P. Assis ${ }^{72}$, G. Avila ${ }^{11}$, A.M. Badescu ${ }^{75}$, A. Bakalova ${ }^{32}$, A. Balaceanu ${ }^{73}$, F. Barbato ${ }^{45,46}$, R.J. Bar-

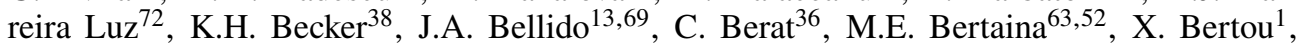
P.L. Biermann ${ }^{b}$, V. Binet ${ }^{6}$, K. Bismark ${ }^{39,8}$, T. Bister ${ }^{42}$, J. Biteau ${ }^{37}$, J. Blazek ${ }^{32}$, C. Bleve ${ }^{36}$, M. Boháčová ${ }^{32}$, D. Bonciolii ${ }^{57,46}$ C. Bonifazi ${ }^{9,26}$, L. Bonneau Arbeletche ${ }^{21}$, N. Borodai ${ }^{70}$, AUGER A.M. Botti ${ }^{8}$, J. Brack $^{d}$, T. Bretz ${ }^{42}$, P.G. Brichetto Orchera ${ }^{8}$, F.L. Briechle ${ }^{42}$, P. Buchholz ${ }^{44}$, A. Bueno ${ }^{78}$, S. Buitink ${ }^{15}$, M. Buscemi ${ }^{47}$, M. Büsken ${ }^{39,8}$, K.S. Caballero-Mora ${ }^{66}$, L. Caccianiga ${ }^{59,49}$, F. Canfora ${ }^{80,81}$, I. Caracas ${ }^{38}$, J.M. Carceller ${ }^{78}$, R. Caruso ${ }^{58,47}$, A. Castellina ${ }^{54,52}$, F. Catalani ${ }^{19}$, G. Cataldi ${ }^{48}$, L. $\operatorname{Cazon}^{72}$, M. Cerda ${ }^{10}$, J.A. Chinellato ${ }^{22}$, J. Chudoba ${ }^{32}$, L. Chytka ${ }^{33}$, R.W. Clay ${ }^{13}$, A.C. Cobos Cerutti ${ }^{7}$, R. Colalillo ${ }^{60,50}$, A. Coleman ${ }^{93}$, M.R. Coluccia $^{48}$, R. Conceição ${ }^{72}$, A. Condorellii ${ }^{45,46}$, G. Consolati ${ }^{49,55}$, F. Contreras ${ }^{11}$, F. Convenga ${ }^{56,48}$, D. Correia dos Santos ${ }^{28}$, C.E. Covault ${ }^{85}$, S. Dasso ${ }^{5,3}$, K. Daumiller ${ }^{41}$, B.R. Dawson ${ }^{13}$, J.A. Day ${ }^{13}$, R.M. de Almeida ${ }^{28}$, J. de Jesús ${ }^{8,41}$, S.J. de Jong ${ }^{80,81}$, G. De Mauro ${ }^{80,81}$, J.R.T. de Mello Neto ${ }^{26,27}$, I. De Mitri ${ }^{45,46}$, J. de Oliveira ${ }^{18}$, D. de Oliveira Franco ${ }^{22}$, F. de Palma ${ }^{56,48}$, V. de Souza ${ }^{20}$, E. De Vito ${ }^{56,48}$, M. del Río ${ }^{11}$, O. Deligny ${ }^{34}$, L. Deval ${ }^{41,8}$, A. di Matteo $^{52}$, C. Dobrigkeit ${ }^{22}$, J.C. D'Olivo ${ }^{68}$, L.M. Domingues Mendes ${ }^{72}$, R.C. dos Anjos ${ }^{25}$, D. dos Santos $^{28}$, M.T. Dova ${ }^{4}$, J. Ebr ${ }^{32}$, R. Engel ${ }^{39,41}$, I. Epicoco ${ }^{56,48}$, M. Erdmann ${ }^{42}$, C.O. Escobar ${ }^{a}$, A. Etchegoyen ${ }^{8,12}$, H. Falcke ${ }^{80,82,81}$, J. Farmer ${ }^{2}$, G. Farrar ${ }^{90}$ A.C. Fauth ${ }^{22}$, N. Fazzini ${ }^{a}$, F. Feldbusch ${ }^{40}$, F. Fenu ${ }^{54,52}$, B. Fick ${ }^{89}$, J.M. Figueira ${ }^{8}$, A. Filipčič $\check{c}^{77,76}$, T. Fitoussi ${ }^{41}$, T. Fodran ${ }^{80}$, M.M. Freire ${ }^{6}$, T. Fujii ${ }^{92, e}$, A. Fuster ${ }^{8,12}$, C. Galea ${ }^{80}$, C. Galelli59,49, B. García ${ }^{7}$, A.L. Garcia Vegas ${ }^{42}$, H. Gemmeke ${ }^{40}$, F. Gesualdi ${ }^{8,41}$, A. Gherghel-Lascu ${ }^{73}$, P.L. Ghia ${ }^{34}$, U. Giaccari ${ }^{80}$, M. Giammarchi ${ }^{49}$, J. Glombitza ${ }^{42}$, F. Gobbi ${ }^{10}$, F. Gollan ${ }^{8}$, G. Golup ${ }^{1}$, M. Gómez Berisso ${ }^{1}$, P.F. Gómez Vitale ${ }^{11}$, J.P. Gongora ${ }^{11}$, J.M. González ${ }^{1}$, N. González ${ }^{14}$, I. Goos ${ }^{1,41}$, D. Góra ${ }^{70}$, A. Gorgi ${ }^{54,52}$, M. Gottowik ${ }^{38}$, T.D. Grubb ${ }^{13}$, F. Guarino ${ }^{60,50}$, G.P. Guedes ${ }^{23}$, E. Guido ${ }^{52,63}$, S. Hahn ${ }^{41,8}$, P. Hamal ${ }^{32}$, M.R. Hampel ${ }^{8}$, P. Hansen ${ }^{4}$, D. Harari ${ }^{1}$, V.M. Harvey ${ }^{13}$, A. Haungs ${ }^{41}$, T. Hebbeker ${ }^{42}$, D. Heck ${ }^{41}$, G.C. Hill ${ }^{13}$, C. Hojvat ${ }^{a}$, J.R. Hörandel ${ }^{80,81}$, P. Horvath ${ }^{33}$, M. Hrabovský ${ }^{33}$, T. Huege ${ }^{41,15}$, A. Insolia ${ }^{58,47}$, P.G. Isar ${ }^{74}$, P. Janecek ${ }^{32}$, J.A. Johnsen ${ }^{86}$, J. Jurysek ${ }^{32}$, A. Kääpä ${ }^{38}$, K.H. Kampert ${ }^{38}$, N. Karastathis ${ }^{41}$, B. Keilhauer ${ }^{41}$, J. Kemp ${ }^{42}$, A. Khakurdikar ${ }^{80}$, V.V. Kizakke Covilakam $^{8,41}$, H.O. Klages ${ }^{41}$, M. Kleifges ${ }^{40}$, J. Kleinfeller ${ }^{10}$, M. Köpke ${ }^{39}$, N. Kunka ${ }^{40}$, B.L. Lago ${ }^{17}$, R.G. Lang ${ }^{20}$, N. Langner ${ }^{42}$, M.A. Leigui de Oliveira ${ }^{24}$, V. Lenok $^{41}$, A. Letessier-Selvon ${ }^{35}$, I. LhenryYvon $^{34}$, D. Lo Presti ${ }^{58,47}$, L. Lopes ${ }^{72}$, R. López ${ }^{64}$, L. Lu ${ }^{94}$, Q. Luce ${ }^{39}$, J.P. Lundquist ${ }^{76}$, A. Machado Payeras $^{22}$, G. Mancarella ${ }^{56,48}$, D. Mandat ${ }^{32}$, B.C. Manning ${ }^{13}$, J. Manshanden ${ }^{43}$, P. Mantsch ${ }^{a}$, S. Marafico ${ }^{34}$, A.G. Mariazzi ${ }^{4}$, I.C. Mariş ${ }^{14}$, G. Marsella ${ }^{61,47}$, D. Martello ${ }^{56,48}$, S. Martinelliili,8, O. Martínez Bravo ${ }^{64}$, M. Mastrodicasa ${ }^{57,46}$, H.J. Mathes ${ }^{41}$, J. Matthews ${ }^{88}$, G. Matthiae ${ }^{62,51}$, E. Mayotte ${ }^{38}$, P.O. Mazur ${ }^{a}$, G. MedinaTanco $^{68}$, D. Melo $^{8}$, A. Menshikov ${ }^{40}$, K.-D. Merenda ${ }^{86}$, S. Michal ${ }^{33}$, M.I. Micheletti ${ }^{6}$, L. Miramonti ${ }^{59,49}$, S. Mollerach ${ }^{1}$, F. Montanet ${ }^{36}$, C. Morello ${ }^{54,52}$, M. Mostafáa ${ }^{91}$, A.L. Müller ${ }^{8}$, M.A. Muller ${ }^{22}$, K. Mulrey ${ }^{15}$, R. Mussa ${ }^{52}$, M. Muzio ${ }^{90}$, W.M. Namasaka ${ }^{38}$, A. Nasr-Esfahani ${ }^{38}$, L. Nellen $^{68}$, M. Niculescu-Oglinzanu ${ }^{73}$, M. Niechciol ${ }^{44}$, D. Nitz ${ }^{89}$, D. Nosek ${ }^{31}$, V. Novotny ${ }^{31}$, L. Nožka ${ }^{33}$, A Nucita $^{56,48}$, L.A. Núñez ${ }^{30}$, M. Palatka ${ }^{32}$, J. Pallotta ${ }^{2}$, P. Papenbreer ${ }^{38}$, G. Parente ${ }^{79}$, A. Parra ${ }^{64}$, J. Pawlowsky ${ }^{38}$, M. Pech ${ }^{32}$, F. Pedreira ${ }^{79}$, J. Pȩkala ${ }^{70}$, R. Pelayo ${ }^{65}$, J. Peña-Rodriguez ${ }^{30}$, E.E. Pereira Martins $s^{39,8}$, J. Perez Armand ${ }^{21}$, C. Pérez Bertolli $^{8,41}$, M. Perlin 8,41 , L. Perrone ${ }^{56,48}$, S. Petrera ${ }^{45,46}$, T. Pierog ${ }^{41}$, M. Pimenta ${ }^{72}$, V. Pirronello ${ }^{58,47}$, M. Platino ${ }^{8}$, B. Pont ${ }^{80}$, M. Pothast ${ }^{81,80}$, P. Privitera ${ }^{92}$, M. Prouza ${ }^{32}$, A. Puyleart ${ }^{89}$, S. Querchfeld ${ }^{38}$, J. Rautenberg ${ }^{38}$, D. Ravignani ${ }^{8}$, M. Reininghaus ${ }^{41,8}$, J. Ridky ${ }^{32}$, F. Riehn ${ }^{72}$, M. Risse ${ }^{44}$, V. Rizi ${ }^{57,46}$, W. Rodrigues de Carvalho ${ }^{21}$, J. Rodriguez Rojo ${ }^{11}$, M.J. Roncoroni ${ }^{8}$, S. Rossoni ${ }^{43}$, M. Roth ${ }^{41}$, E. Roulet ${ }^{\text {', }}$ A.C. Rovero ${ }^{5}$, P. Ruehl ${ }^{44}$, A. Saftoiu ${ }^{73}$, F. Salamida ${ }^{57,46}$, H. Salazar ${ }^{64}$, G. Salina ${ }^{51}$, J.D. Sanabria Gomez ${ }^{30}$, F. Sánchez ${ }^{8}$, E.M. Santos ${ }^{21}$, E. Santos ${ }^{32}$, F. Sarazin ${ }^{86}$, R. Sarmento ${ }^{72}$, C. Sarmiento-Cano ${ }^{8}$, R. Sato ${ }^{11}$, P. Savina ${ }^{56,48,34,94}$, C.M. Schäfer ${ }^{41}$, V. Scherini ${ }^{56,48}$, H. Schieler ${ }^{41}$, M. Schimassek ${ }^{39,8}$, M. Schimp ${ }^{38}$, F. Schlüter ${ }^{41,8}$, D. Schmidt ${ }^{39}$, O. Scholten ${ }^{84,15}$, P. Schovánek ${ }^{32}$, F.G. Schröder ${ }^{93,41}$, S. Schröder ${ }^{38}$, J. Schulte ${ }^{42}$, S.J. Sciutto ${ }^{4}$, M. Scornavacche ${ }^{8,41}$, A. Segreto ${ }^{53,47}$, S. Sehgal ${ }^{38}$, R.C. Shellard ${ }^{16}$, G. Sigl ${ }^{43}$, G. Silli ${ }^{8,41}$, O. Sima ${ }^{73, f}$, R. Šmída ${ }^{92}$, P. Sommers ${ }^{91}$, J.F. Soriano ${ }^{87}$, J. Souchard ${ }^{36}$, R. Squartini ${ }^{10}$, M. Stadelmaier ${ }^{41,8}$, D. Stanca ${ }^{73}$, S. Staničc ${ }^{76}$, J. Stasielak ${ }^{70}$, P. Stassi ${ }^{36}$, A. Streich ${ }^{39,8}$, M. Suárez-Durán ${ }^{14}$, T. Sudholz ${ }^{13}$, T. Suomijärvi ${ }^{37}$, A.D. Supanitsky ${ }^{8}$, Z. Szadkowski ${ }^{71}$, A. Tapia ${ }^{29}$, C. Taricco ${ }^{63,52}$, C. Timmermans ${ }^{81,80}$, O. Tkachenko ${ }^{41}$, P. Tobiska ${ }^{32}$, C.J. Todero Peixoto ${ }^{19}$, B. Tomé ${ }^{72}$, Z. Torrès ${ }^{36}$, A. Travaini ${ }^{10}$, P. Travnicek ${ }^{32}$, 


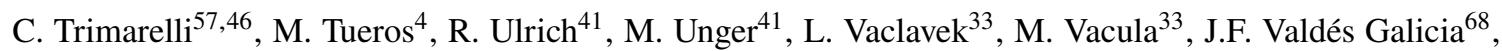
L. Valore ${ }^{60,50}$, E. Varela ${ }^{64}$, A. Vásquez-Ramírez ${ }^{30}$, D. Veberič ${ }^{41}$, C. Ventura ${ }^{27}$, I.D. Vergara Quispe $^{4}$,

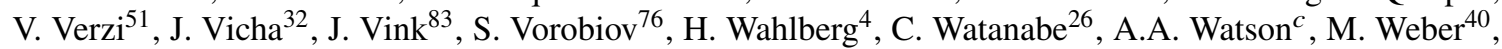
A. Weindl ${ }^{41}$, L. Wiencke ${ }^{86}$, H. Wilczyński ${ }^{70}$, M. Wirtz ${ }^{42}$, D. Wittkowski ${ }^{38}$, B. Wundheiler ${ }^{8}$, A. Yushkov $^{32}$, O. Zapparrata ${ }^{14}$, E. Zas ${ }^{79}$, D. Zavrtanik ${ }^{76,77}$, M. Zavrtanik ${ }^{77,76}$, L. Zehrer ${ }^{76}$

${ }^{1}$ Centro Atómico Bariloche and Instituto Balseiro (CNEA-UNCuyo-CONICET), San Carlos de Bariloche, Argentina

${ }^{2}$ Centro de Investigaciones en Láseres y Aplicaciones, CITEDEF and CONICET, Villa Martelli, Argentina

${ }^{3}$ Departamento de Física and Departamento de Ciencias de la Atmósfera y los Océanos, FCEyN, Universidad de Buenos Aires and CONICET, Buenos Aires, Argentina

${ }^{4}$ IFLP, Universidad Nacional de La Plata and CONICET, La Plata, Argentina

${ }^{5}$ Instituto de Astronomía y Física del Espacio (IAFE, CONICET-UBA), Buenos Aires, Argentina

${ }^{6}$ Instituto de Física de Rosario (IFIR) - CONICET/U.N.R. and Facultad de Ciencias Bioquímicas y Farmacéuticas U.N.R., Rosario, Argentina

${ }^{7}$ Instituto de Tecnologías en Detección y Astropartículas (CNEA, CONICET, UNSAM), and Universidad Tecnológica Nacional - Facultad Regional Mendoza (CONICET/CNEA), Mendoza, Argentina

${ }^{8}$ Instituto de Tecnologías en Detección y Astropartículas (CNEA, CONICET, UNSAM), Buenos Aires, Argentina

${ }^{9}$ International Center of Advanced Studies and Instituto de Ciencias Físicas, ECyT-UNSAM and CONICET, Campus Miguelete - San Martín, Buenos Aires, Argentina

${ }^{10}$ Observatorio Pierre Auger, Malargüe, Argentina

11 Observatorio Pierre Auger and Comisión Nacional de Energía Atómica, Malargüe, Argentina

12 Universidad Tecnológica Nacional - Facultad Regional Buenos Aires, Buenos Aires, Argentina

${ }^{13}$ University of Adelaide, Adelaide, S.A., Australia

${ }^{14}$ Université Libre de Bruxelles (ULB), Brussels, Belgium

15 Vrije Universiteit Brussels, Brussels, Belgium

${ }^{16}$ Centro Brasileiro de Pesquisas Fisicas, Rio de Janeiro, RJ, Brazil

${ }^{17}$ Centro Federal de Educação Tecnológica Celso Suckow da Fonseca, Nova Friburgo, Brazil

18 Instituto Federal de Educação, Ciência e Tecnologia do Rio de Janeiro (IFRJ), Brazil

${ }^{19}$ Universidade de São Paulo, Escola de Engenharia de Lorena, Lorena, SP, Brazil

${ }^{20}$ Universidade de São Paulo, Instituto de Física de São Carlos, São Carlos, SP, Brazil

${ }^{21}$ Universidade de São Paulo, Instituto de Física, São Paulo, SP, Brazil

${ }^{22}$ Universidade Estadual de Campinas, IFGW, Campinas, SP, Brazil

${ }^{23}$ Universidade Estadual de Feira de Santana, Feira de Santana, Brazil

${ }^{24}$ Universidade Federal do ABC, Santo André, SP, Brazil

25 Universidade Federal do Paraná, Setor Palotina, Palotina, Brazil

${ }^{26}$ Universidade Federal do Rio de Janeiro, Instituto de Física, Rio de Janeiro, RJ, Brazil

27 Universidade Federal do Rio de Janeiro (UFRJ), Observatório do Valongo, Rio de Janeiro, RJ, Brazil

${ }^{28}$ Universidade Federal Fluminense, EEIMVR, Volta Redonda, RJ, Brazil

${ }^{29}$ Universidad de Medellín, Medellín, Colombia

${ }^{30}$ Universidad Industrial de Santander, Bucaramanga, Colombia

${ }^{31}$ Charles University, Faculty of Mathematics and Physics, Institute of Particle and Nuclear Physics, Prague, Czech Republic

32 Institute of Physics of the Czech Academy of Sciences, Prague, Czech Republic

${ }^{33}$ Palacky University, RCPTM, Olomouc, Czech Republic

${ }^{34}$ CNRS/IN2P3, IJCLab, Université Paris-Saclay, Orsay, France

${ }^{35}$ Laboratoire de Physique Nucléaire et de Hautes Energies (LPNHE), Sorbonne Université, Université de Paris, CNRSIN2P3, Paris, France

${ }^{36}$ Univ. Grenoble Alpes, CNRS, Grenoble Institute of Engineering Univ. Grenoble Alpes, LPSC-IN2P3, 38000 Grenoble, France

37 Université Paris-Saclay, CNRS/IN2P3, IJCLab, Orsay, France

38 Bergische Universität Wuppertal, Department of Physics, Wuppertal, Germany

${ }^{39}$ Karlsruhe Institute of Technology (KIT), Institute for Experimental Particle Physics, Karlsruhe, Germany

${ }^{40}$ Karlsruhe Institute of Technology (KIT), Institut für Prozessdatenverarbeitung und Elektronik, Karlsruhe, Germany

${ }^{41}$ Karlsruhe Institute of Technology (KIT), Institute for Astroparticle Physics, Karlsruhe, Germany

42 RWTH Aachen University, III. Physikalisches Institut A, Aachen, Germany

43 Universität Hamburg, II. Institut für Theoretische Physik, Hamburg, Germany

${ }^{44}$ Universität Siegen, Department Physik - Experimentelle Teilchenphysik, Siegen, Germany 
${ }^{45}$ Gran Sasso Science Institute, L'Aquila, Italy

46 INFN Laboratori Nazionali del Gran Sasso, Assergi (L’Aquila), Italy

${ }^{47}$ INFN, Sezione di Catania, Catania, Italy

${ }^{48}$ INFN, Sezione di Lecce, Lecce, Italy

${ }^{49}$ INFN, Sezione di Milano, Milano, Italy

${ }^{50}$ INFN, Sezione di Napoli, Napoli, Italy

51 INFN, Sezione di Roma "Tor Vergata", Roma, Italy

52 INFN, Sezione di Torino, Torino, Italy

53 Istituto di Astrofisica Spaziale e Fisica Cosmica di Palermo (INAF), Palermo, Italy

54 Osservatorio Astrofisico di Torino (INAF), Torino, Italy

55 Politecnico di Milano, Dipartimento di Scienze e Tecnologie Aerospaziali , Milano, Italy

56 Università del Salento, Dipartimento di Matematica e Fisica "E. De Giorgi”, Lecce, Italy

${ }^{57}$ Università dell'Aquila, Dipartimento di Scienze Fisiche e Chimiche, L'Aquila, Italy

${ }^{58}$ Università di Catania, Dipartimento di Fisica e Astronomia, Catania, Italy

${ }^{59}$ Università di Milano, Dipartimento di Fisica, Milano, Italy

${ }^{60}$ Università di Napoli "Federico II", Dipartimento di Fisica "Ettore Pancini”, Napoli, Italy

${ }^{61}$ Università di Palermo, Dipartimento di Fisica e Chimica ”E. Segrè”, Palermo, Italy

62 Università di Roma “Tor Vergata”, Dipartimento di Fisica, Roma, Italy

63 Università Torino, Dipartimento di Fisica, Torino, Italy

64 Benemérita Universidad Autónoma de Puebla, Puebla, México

65 Unidad Profesional Interdisciplinaria en Ingeniería y Tecnologías Avanzadas del Instituto Politécnico Nacional (UPIITA-IPN), México, D.F., México

66 Universidad Autónoma de Chiapas, Tuxtla Gutiérrez, Chiapas, México

${ }^{67}$ Universidad Michoacana de San Nicolás de Hidalgo, Morelia, Michoacán, México

${ }^{68}$ Universidad Nacional Autónoma de México, México, D.F., México

${ }^{69}$ Universidad Nacional de San Agustin de Arequipa, Facultad de Ciencias Naturales y Formales, Arequipa, Peru

${ }^{70}$ Institute of Nuclear Physics PAN, Krakow, Poland

${ }^{71}$ University of Łódź, Faculty of High-Energy Astrophysics,Łódź, Poland

${ }^{72}$ Laboratório de Instrumentação e Física Experimental de Partículas - LIP and Instituto Superior Técnico - IST, Universidade de Lisboa - UL, Lisboa, Portugal

73 "Horia Hulubei” National Institute for Physics and Nuclear Engineering, Bucharest-Magurele, Romania

${ }^{74}$ Institute of Space Science, Bucharest-Magurele, Romania

75 University Politehnica of Bucharest, Bucharest, Romania

${ }^{76}$ Center for Astrophysics and Cosmology (CAC), University of Nova Gorica, Nova Gorica, Slovenia

${ }^{77}$ Experimental Particle Physics Department, J. Stefan Institute, Ljubljana, Slovenia

${ }^{78}$ Universidad de Granada and C.A.F.P.E., Granada, Spain

${ }^{79}$ Instituto Galego de Física de Altas Enerxías (IGFAE), Universidade de Santiago de Compostela, Santiago de Compostela, Spain

${ }^{80}$ IMAPP, Radboud University Nijmegen, Nijmegen, The Netherlands

${ }^{81}$ Nationaal Instituut voor Kernfysica en Hoge Energie Fysica (NIKHEF), Science Park, Amsterdam, The Netherlands

${ }^{82}$ Stichting Astronomisch Onderzoek in Nederland (ASTRON), Dwingeloo, The Netherlands

${ }^{83}$ Universiteit van Amsterdam, Faculty of Science, Amsterdam, The Netherlands

${ }^{84}$ University of Groningen, Kapteyn Astronomical Institute, Groningen, The Netherlands

${ }^{85}$ Case Western Reserve University, Cleveland, OH, USA

${ }^{86}$ Colorado School of Mines, Golden, CO, USA

87 Department of Physics and Astronomy, Lehman College, City University of New York, Bronx, NY, USA

${ }^{88}$ Louisiana State University, Baton Rouge, LA, USA

${ }^{89}$ Michigan Technological University, Houghton, MI, USA

${ }^{90}$ New York University, New York, NY, USA

${ }^{91}$ Pennsylvania State University, University Park, PA, USA

92 University of Chicago, Enrico Fermi Institute, Chicago, IL, USA

93 University of Delaware, Department of Physics and Astronomy, Bartol Research Institute, Newark, DE, USA

94 University of Wisconsin-Madison, Department of Physics and WIPAC, Madison, WI, USA

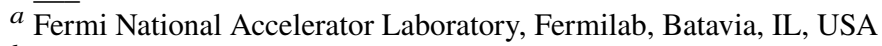

${ }^{b}$ Max-Planck-Institut für Radioastronomie, Bonn, Germany

${ }^{c}$ School of Physics and Astronomy, University of Leeds, Leeds, United Kingdom

${ }^{d}$ Colorado State University, Fort Collins, CO, USA

$e^{e}$ now at Hakubi Center for Advanced Research and Graduate School of Science, Kyoto University, Kyoto, Japan 
$f$ also at University of Bucharest, Physics Department, Bucharest, Romania 